\title{
Relationship Between PET/CT Response and Survival in Patients with Non-Small-Cell Lung Cancer Treated with Definitive Chemoradiotherapy
}

\author{
Sureyya SARIHAN ${ }^{1}$, Gokce Belge BILGIN ${ }^{2}$, Deniz SIGIRLI ${ }^{3}$ \\ ${ }^{1}$ Uludag University, Faculty of Medicine, Department of Radiation Oncology \\ ${ }^{2}$ Uludag University, Faculty of Medicine, Department of Nuclear Medicine \\ ${ }^{3}$ Uludag University, Faculty of Medicine, Department of Biostatistics, Bursa, TURKEY
}

\begin{abstract}
We aimed to evaluate the relationship between PET/CT response and survival in patients with non-small cell lung cancer (NSCLC) treated with curative chemoradiotherapy. Between January and December 2012, 51 patients were treated. The median age was 61 (29-79) and the M/F ratio was 46/5. Eighty two percent of the cases were stage III and $53 \%$ were squamous cell carcinoma. Median 6300 cGy (4860-7525) radiotherapy delivered and 92\% of patients received chemotherapy. The median follow-up was 27 months (7-96 months) in November 2019. The objective response was 71\% with CT at 1 month and 76\% with PET/CT at 3 months. There was a significant correlation between response-1 and response-3 $(p<0.001)$. Tumor SUVmean $<2.81$, SUVmax change $\geq 70 \%$ was associated with response-1 ( $p<0.05)$. The median and 5-year overall (OS) and progression-free (PFS) survival rates were 54 months, 40\% and 35 months, 38\%, respectively. In Cox model, for each 1 unit increase, SUVmeanbase (HR: 1.18, 95\% Cl: 1.01-1.38) and SUVmean3 (HR: 2.65, 95\% Cl: 1.24-5.66) were found unfavorable factors for OS, whereas SUVmean3 (HR: 2.01, 95\% Cl: 1.02-3.93) was also found to be a poor prognostic factor for PFS. PET/CT parameters can be used as useful markers for prognosis in patients with NSCLC undergoing curative chemoradiotherapy. It is believed that early assessment during and after treatment can be advantageous in terms of treatment modification.
\end{abstract}

Keywords: Non-small cell lung cancer, Definitive chemo-RT, PET/CT response, Survival

\section{INTRODUCTION}

The current treatment approach for locally advanced non-small-cell lung cancer (NSCLC) is 60-Gy radiotherapy (RT) concomitant with chemotherapy (CHE), and local recurrence rates vary between $15 \%$ and $40 \% .^{1,2,3}$ In the randomised study by Perez et al., local control (LC) was shown to be associated with the dose of RT and survival. ${ }^{4}$ However, this study included a response evaluation based on chest radiography and reported that 3-year overall survival (OS) for complete response (CR), partial response (PR) and stable response were $23 \%, 10 \%$ and $5 \%$, respectively.

The use of computed tomography (CT) in response evaluation and restaging has been a major develop- ment. ${ }^{5}$ Imaging with CT provides anatomical information based on tumour diameter, and it was reported that the response could be seen for up to 11 months. ${ }^{6}$ Increased glucose transport in malignant tissues was first described by Warburg, and it was believed that the biochemical/metabolic changes associated with treatment could be used as a marker of response. ${ }^{7}$ Imaging with positron emission tomography (PET) using radioisotope-labelled glucose tracer $18 \mathrm{~F}$-fluorodeoxyglucose (18F-FDG) was shown to detect lesions larger than $5 \mathrm{~mm}$ with high accuracy. ${ }^{8}$ In addition to the metabolic visual correlation that it provides, integration of CT with PET (PET/CT) can also evaluate tumour and nodal spread more accurately. ${ }^{9}$ 
PET/CT was proven to be superior to CT in terms of diagnosis, staging, RT-planning, response evaluation and survival estimation in patients with lung tumours. ${ }^{10-13}$ In a systematic review, it was reported that the clinical CR, PR and stable response rate was $35 \%, 35 \%$ and $30 \%$ with CT evaluation following induction therapy in stage (s) II patients while pathological (p) CR was 20\%, $5 \%$ and $3 \%$ for these responses, respectively. ${ }^{14}$ In this study, although the false negative (FN) and false positive (FP) rates for tumor were $60 \%$ and $31 \%$ with CT whereas these rates were $0 \%$ and $5 \%$, respectively, if the metabolic response in PET was $>80 \%$. In terms of nodal response, FN rates were reported as $33 \%$ and $25 \%$ for CT and PET, respectively.

Maximum standardised uptake value (SUVmax) is widely used to predict the response and prognosis with PET/CT. In the meta-analysis, the SUVmax measured at diagnosis or a median week 12 (824) following RT was reported to be significant in terms of LC and OS with a minimum cut-off value of $\geq 5 .{ }^{15}$ It was underlined that the SUV change should at least be $\geq 30 \%$ for response. ${ }^{16}$ Cerfolio et al. reported that a decrease of $\geq 80 \%$ in SUVmax in the first month after treatment was found to be superior than the change in size on CT, and the sensitivity, specificity and accuracy for pCR were reported to be $90 \%, 100 \%$ and $96 \%$, respectively. ${ }^{17}$ Eschmann et al. used PET/CT three times to evaluate 65 patients prior to treatment, at 2 weeks following the neoadjuvant $\mathrm{CHE}$ and at 2 weeks after completion of sequential RT. ${ }^{18}$ This study showed that in patients who exhibited a SUVmax decrease of $>60 \%$ following neoadjuvant CHE, 5-year OS was significantly increased (60\% vs 15\%). On the other hand, the cut-off value was reported to be $75 \%$ for a survival difference following RT. In another study by Eschmann et al. that included 70 sIII patients treated with neoadjuvant chemo-RT, the sensitivity, specificity and accuracy of PET/ CT in detecting residual tumours were $94 \%, 80 \%$ and $91 \%$ whereas these values were $77 \%, 68 \%$ and $73 \%$, respectively in detecting nodal involvement. ${ }^{19}$ This study underlines that a decrease of $\geq$ $80 \%$ in SUV value is the best prognostic factor (PF) in terms of outcomes, and surgery does not change the outcome in patients with metabolic progression. In the ACRIN6668/RTOG 0235 study that included 173 patients with NSCLC who were administered curative chemo-RT, it was shown that the 2-year OS was significantly increased $(47 \%$ vs $25 \%$ ) when SUVmax was $\leq 5$ at 3 months after treatment. ${ }^{20}$

This study aimed to evaluate the relationship between PET/CT response and survival in patients with NSCLC who were administered curative chemo-RT and to review the relevant literature.

\section{PATIENTS and METHODS}

We retrospectively analysed the data of 51 patients treated with curative chemo-RT between 1 January and 31 December 2012 in our department (Table 1). Written informed consent obtained from all patients. The current study was approved by the Institutional Ethics Committee.

$\mathrm{CT}, \mathrm{PET} / \mathrm{CT}$ and cranial magnetic resonance imaging were used for staging cancer in all patients. Radiotherapy was applied using LINAC with three-dimensional conformal RT (3DCRT) or intensity-modulated RT (IMRT). Cisplatin-based multiagent CHE (paclitaxel, docetaxel or gemcitabine) was administered using the standard regimen. In patients receiving weekly concomitant $\mathrm{CHE}$, the number of cycles was calculated as the standard CHE equivalent of the cumulative dose. Comorbidity status was assessed according to the pulmonary, cardiovascular, renal, neurological and endocrine functions.

Responses were assessed at month 1 using CT (response-1), at month 3 using PET/CT (response-3) and at month 6 using CT or PET/CT (response-6) after RT. The patients underwent PET/CT following at least 4 hours of fasting. All PET/CT images performed in our institution was evaluated one by one by the relevant author (GBB) in November 2019. The volume of interest (VOI) that represents the highest radioactivity of the lesion in the $18 \mathrm{~F}$ FDG PET/CT images was determined semi-automatically. SUVmean (i.e. the mean of the SUV values), and SUVmax that represents the highest SUV value within the identified VOI were calculated. The threshold value of metabolic tumour volume (MTV) was determined to be $42 \%$ of the lesion SUVmax and was automatically calculated. Total lesion glycolysis (TLG) was calculated by multiplying the MTV and SUV mean values. Toxicity was evaluated according to the Common Terminology 
Table 1. Demografic features and treatment parameters

Demografic features and treatment $\quad \mathrm{n}$ (range/ \%)

parameters $(n=51)$

Age (median, year)

$61(29-79)$

Male/Female

46 (90) / 5 (10)

KPS (median)

$90(70-100)$

Smoking history / median pack/year

Tumour size (median, $\mathrm{cm}$ )

$45(88) / 45(7.5-120)$

$5.5(1.4-14)$

Symptom time (median, month) $(n=30) \quad 3(1-24)$

T stage (AJCC 2010)

T1 2 (4)

T2 $20(39)$

T3 $12(23)$

T4 17 (33)

TNM staging

$1 \quad 1(2)$

II $5(10)$

IIIA $28(55)$

IIIB $14(27)$

IV $3(6)$

RT dose (median, cGy)

RT fraction dose (median, cGy)

RT duration (median, day)

RT type - IMRT/ Conformal

Treatment break (median, day) $(n=18)$

Neoadjuvant CHE / median cycles

Concurrent $\mathrm{CHE} /$ median cycles

Adjuvant CHE / median cycles

Total CHE / median cycles

Family cancer history

Comorbidity

Second primary

GTV median (cc) ( $n=44)$

CTV median (cc) ( $n=42)$

MLD median (Gy) $(n=43)$

PET/CT parameters

SUVmaxbase (median) $(n=47)$

SUVmeanbase (median) $(n=29)$

MTVbase (median) $(n=29)$

TLGbase (median) $(n=29)$

SUVmax3 (median) ( $n=38)$

SUVmean3 (median) $(n=20)$

MTV3 (median) $(n=20)$

TLG3 (median) $(n=20)$

SUVmax, change \% (median) $(n=38)$

SUVmean, change \% (median) $(n=16)$

MTV, change \% (median) $(n=12)$

TLG, change \% (median) $(n=13)$
6300 (4860-7525)

180 (180-250)

$52(41-65)$

$14(27) / 37(73)$

$4(1-20)$

$21(41) / 4(1-7)$

$36(70) / 3(1-4)$

$29(57) / 3(1-6)$

$47(92) / 6(1-10)$

17 (33)

25 (49)

$6(12)$

$181.7(10.15-993.73)$

784.09 (184-1850.63)

$19,75(8.75-28.21)$

$15.25(2.5-31.5)$

$9.37(2.23-20.32)$

$29.38(4.04-129)$

$281.94(12.8-1141.85)$

$3.6(0-13.2)$

$2.81(1.38-5.47)$

$15.14(2.3-215)$

$41.83(8.89-1058.25)$

$81 \%(3-100 \%)$

$65 \%(11-90 \%)$

$63 \%(3-92 \%)$

$85 \%(40-96 \%)$

Criteria for Adverse Events v4. ${ }^{21}$ The patients with local or distant recurrence during follow-up were administered CHE, targeted therapy, palliative RT or supportive care.

Statistical analysis was conducted using SPSS v.21 in November 2019. Overall survival were calculated from diagnosis until death or last follow-up, and progression-free survival (PFS) was calculated from diagnosis until progression, death or last follow-up. The analysis was performed using median and also various cut-off values were reported in the literature (e.g. $\geq 2.5, \geq 5, \geq 10, \geq 15$ and $30 \%$ $100 \%$ ) for all PET/CT parameters. The correlations between the variables were assessed using Pearson chi-square and Fisher's exact tests. Survival rates were analysed using the Kaplan-Meier method and univariate analysis log-rank test. Cox regression analysis was used for multivariate analysis. A $\mathrm{p}$ value of $<0.05$ was considered statistically significant.

\section{RESULTS}

The clinical characteristics and treatment parameters of the patients are presented in Table 1. The median age was 61 (29-79) years, and the male/ female ratio was $46 / 5$. Overall, $53 \%$ patients were diagnosed with squamous cell carcinoma (SCC), $41 \%$ with adenocarcinoma and $6 \%$ with NSCLC. In total, $82 \%$ of the patients had sIII according to the AJCC 2010 staging and 3 patients were diagnosed with solitary bone or adrenal metastasis. A total of $42 \%$ of the 12 patients who were assessed using mediastinoscopy, mediastinotomy or EBUS were found to be at stage pNO, which was considered clinical N0. The clinical nodal stages of Nx, $\mathrm{N} 0, \mathrm{~N} 1, \mathrm{~N} 2$ and $\mathrm{N} 3$ were found in $10 \%, 16 \%, 4 \%$, $61 \%$ and $10 \%$ of the patients, respectively.

The median symptom duration was 3 (1-24) months. The symptoms at diagnosis were presented in Table 1. In total, 45 patients $(88 \%)$ had a history of smoking. Twenty-five patients (49\%) had comorbidities, and 4 of these (8\%) had a history of surgery. Six (12\%) patients had second primary malignancy prior (prostate, skin, larynx, endometrium and thymic) or subsequent to diagnosis (rectum, at 32 months).

The median 6300 (4860-7525) cGy RT was administered with $180 \mathrm{cGy}(180-250)$ fractions. Treat- 


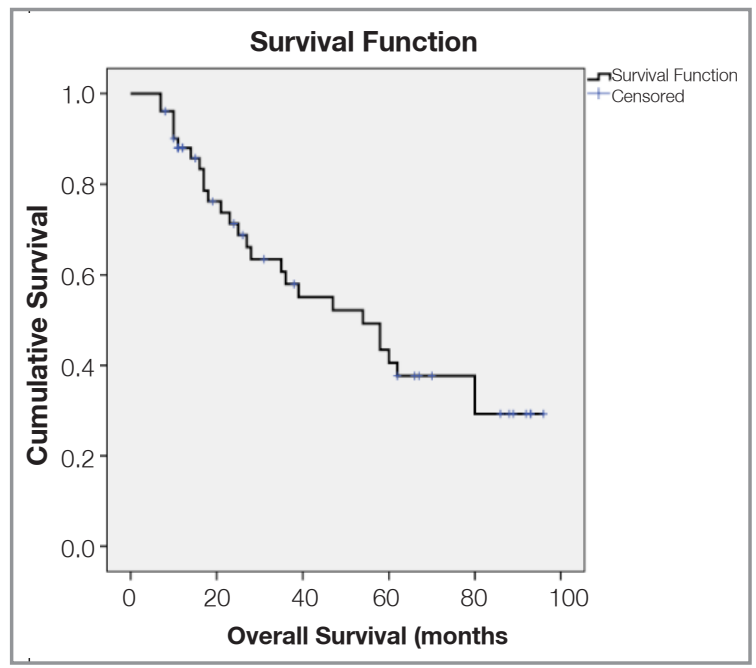

Figure 1. Overall survival

ment was interrupted for a median of 4 days (120 ) in 18 cases due to machine breakdown or G1-3 hematologic, lung and esophageal toxicity during RT. Acute grade (G) 1-3 complications occurred in 38 patients $(75 \%)$. There was no $\geq \mathrm{G} 4$ acute complication. A total of 47 patients $(92 \%)$ were administered a median of 6 CHE cycles (1-10). Five patients received maintenance erlotinib for a median of 36 (2-60) months.

At 1 month after treatment according to CT evaluation, CR, PR and stable response rates were $6 \%$, $65 \%$ and $29 \%$, respectively. In 42 patients who were evaluated using PET/CT on median day of 94 (31-127), CR, PR, stable response and progression rates were found to be $19 \%, 57 \%, 21 \%$ and $2 \%$, respectively. Excluding patients who had undergone surgery or who were not evaluated, there were 27 patients evaluated by CT or PET/CT at 6 months, and $\mathrm{CR}, \mathrm{PR}$, stable response and progression rates were $41 \%, 37 \%, 11 \%$, and $11 \%$, respectively. Total of 8 patients underwent surgery at 6 months, and the $\mathrm{pCR} /$ near-pCR rate $(<10 \%$ residual cells) was $75 \%$ (6/8). During the follow-up, pCR was achieved in 1 of 3 patients who underwent surgery at months 13, 36 and 40, and the total pCR/ near-pCR rate wAS 64\% (7/11). All of the operated patients had clinical N2/3 disease, and the rate of nodal response was $82 \%(9 / 11)$. The median time to operation was 2.5 (1-40) months.

In a median of 3 (3-6) months following the RT, radiation pneumonitis (RP) occurred in $22(43 \%)$

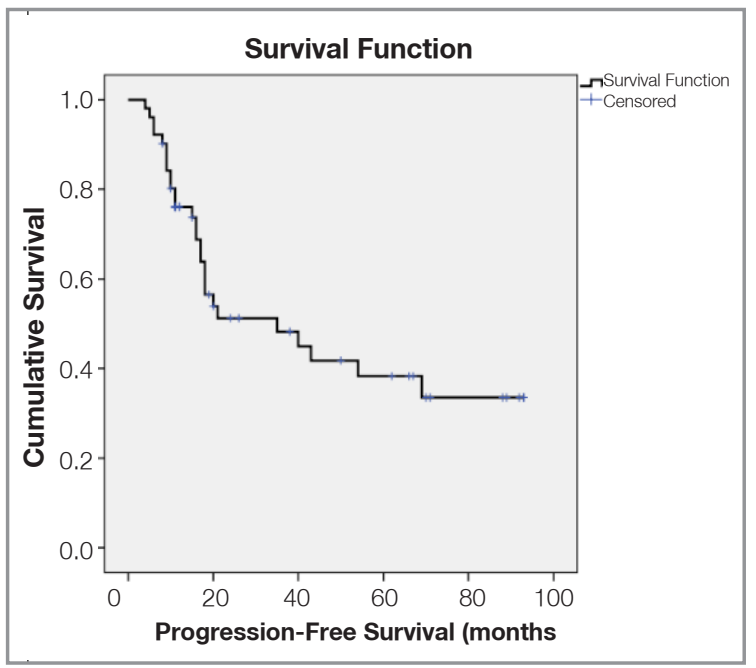

Figure 2. Progression-free survival

patients: G1 in 2 (4\%), G2 in $17(33 \%)$ and G3 in $3(6 \%)$. Pleural effusion was observed in 8 patients (16\%) in a median of $3(3-11)$ months and pericardial effusion/thickening occurred in 2 patients (4\%) in 4 and 7 months.

Locoregional or distant recurrence was observed in 29 patients (57\%). Locoregional recurrences were seen in a median of $9(5-66)$ months in $8 \%(n=4)$ and distant recurrences were seen in a median of 16 (4-69) months in 29\% ( $\mathrm{n}=15)$ patients, respectively. In 10 of patients (20\%), locoregional and distant recurrences concomitantly developed in a median of 17 (9-35) months. Single-organ or multipl metastases occurred in $17(33 \%)$ and 8 patients (16\%), respectively. The most common metastatic sites were bones $(\mathrm{n}=15,29 \%)$ and lungs $(\mathrm{n}=8$, $16 \%)$, respectively.

The median follow-up was 27 (7-96) months from diagnosis. Seven patients (14\%) were alive, 26 $(51 \%)$ were deceased and $18(35 \%)$ were lost to follow-up at the time of evaluation. The causes of death were as follows: multiple organ failure with disease progression (88\%), lung infection $(8 \%)$ and toxic hepatitis (4\%). The median and 5-year OS and PFS rates were 54 months (28-79), 40\% and 35 months (5-64), 38\%, respectively (Figure $1,2)$.

The median values for PET/CT parameters are presented in Table 1. The number of evaluated patients for SUVmax, SUVmean, MTV and TLG was different because there were patients who were tested at 


\begin{tabular}{|c|c|c|c|c|c|c|c|c|c|c|c|c|}
\hline \multirow[t]{2}{*}{ Parameters } & \multicolumn{4}{|c|}{ Response-1 (n: 51) } & \multicolumn{4}{|c|}{ Response-3 ( $n=42)$} & \multicolumn{4}{|c|}{ Response- 6 ( $n=27$ ) } \\
\hline & CR & PR & S/P & p & CR & PR & $\mathbf{S} / \mathbf{P}$ & $p$ & CR & PR & $\mathbf{S} / \mathbf{P}$ & $p$ \\
\hline SUVmax3 & & & & & & & & & & & & 0.030 \\
\hline$<5(\mathrm{n}: 14)$ & & & & & & & & & $90 \%$ & $71 \%$ & $0 \%$ & \\
\hline$\geq 5$ (n: 6) & & & & & & & & & $10 \%$ & $29 \%$ & $100 \%$ & \\
\hline SUVmean3 & & & & & & & & & & & & \\
\hline$<2.81$ (n: 9) & $100 \%$ & $61.5 \%$ & $0 \%$ & 0.014 & & & & & & & & \\
\hline$\geq 2.81$ (n: 11) & $0 \%$ & $38.5 \%$ & $100 \%$ & & & & & & & & & \\
\hline SUVmax, change\% & & & & & & & & & & & & \\
\hline$\geq 70$ (n: 25) & $100 \%$ & $77 \%$ & $\% 30$ & 0.019 & & & & & & & & \\
\hline$<70$ (n: 13) & $0 \%$ & $23 \%$ & $70 \%$ & & & & & & & & & \\
\hline SUVmax, change\% & & & & & & & & & & & & 0.024 \\
\hline$\geq 60$ (n: 13) & & & & & & & & & $90 \%$ & $57 \%$ & $0 \%$ & \\
\hline$<60$ (n: 7) & & & & & & & & & $10 \%$ & $43 \%$ & $100 \%$ & \\
\hline SUVmean3 & 1.90 & 3.30 & 5.74 & 0.037 & & & & & 2.60 & 4.11 & 7.89 & 0.041 \\
\hline & (0-3.80) & $(0-9.73)$ & $(0-13.20)$ & & & & & & $(0-7.80)$ & $(0-9.52)$ & $(7.72-9.73)$ & \\
\hline & (n: 2) & (n: 26) & (n: 10) & & & & & & (n: 10) & (n: 7) & (n: 3) & \\
\hline SUVmax3 & 2.24 & 2.52 & 4.00 & 0.026 & & & & & & & & \\
\hline & & (1.38-5.42) & $(2.81-5.47)$ & & & & & & & & & \\
\hline & $(n: 1)$ & (n: 13) & $(n: 6)$ & & & & & & & & & \\
\hline Symptom time & & & & & & & & 0.013 & & & & \\
\hline$<3$ months $(\mathrm{n}: 11)$ & & & & & $100 \%$ & $31 \%$ & $25 \%$ & & & & & \\
\hline$\geq 3$ months (n: 14) & & & & & $0 \%$ & $69 \%$ & $75 \%$ & & & & & \\
\hline Radiation pneumonitis & & & & & & & & 0.021 & & & & \\
\hline presence (n: 22) & & & & & $62.5 \%$ & $62.5 \%$ & $55.5 \%$ & & & & & \\
\hline absence (n: 20) & & & & & $37.5 \%$ & $37.5 \%$ & $44.5 \%$ & & & & & \\
\hline
\end{tabular}

another center, whose lesions could not be localised after treatment and who could not be evaluated owing to RP.

The variables that affected response were analysed (Table 2). A tumour SUV mean 3 of $<2.81$ and SUVmax change of $\geq 70 \%$ were found to be significantly correlated with response-1 $(\mathrm{p}=0.014, \mathrm{p}=0.019)$. The SUVmax3 and SUVmean3 values for CR, PR and stable response at month 1 were found to be $1.90,3.30$ and $5.74(\mathrm{p}=0.037)$ and 2.24, 2.52 and $4.00(\mathrm{p}=0.026)$, respectively. A duration of symptoms of $<3$ months and presence of RP were significantly correlated with response- $3(\mathrm{p}=0.013, \mathrm{p}=$ 0.021 ), and a SUVmax 3 of $<5$ and SUVmax change of $\geq 60 \%$ were correlated with response- 6 ( $\mathrm{p}=$ 0.030, $\mathrm{p}=0.024)$. SUV $\max 3$ values were 2.6, 4.11 and 7.89 for CR, PR and stable response at month 6 , respectively and were found statistically significant $(\mathrm{p}=0.041)$.
The correlations between all variables were analysed (Table 3). Response- 1 and response- 3 and also response- 3 and response- 6 were significantly correlated each other $(\mathrm{p}<0.001, \mathrm{p}<0.001)$. SUVmaxbase was found to be positively correlated with tumour size, clinical tumour volume (CTV), TLG, SUVmeanbase and negatively correlated with Karnofsky performance status (KPS) $(\mathrm{p}<0.05)$. There was a positive correlation between SUVmax 3 and SUVmean3 $(\mathrm{p}<0.001)$. The rate of RP was higher in patients who were administered a fraction dose of $<200$ cGy (55\% vs $8 \%, p=0.008)$, who received concomitant CHE for $\geq 3$ cycles $(65 \%$ vs $19 \%$, $\mathrm{p}=$ $0.015)$ and who had an objective response (OR) at month $3(62.5 \%$ vs $11 \%, p=0.021)$. The patients who had RP were found to have significantly larger CTV (865 vs $735 \mathrm{cc}, \mathrm{p}=0.039$ ). The patients with a mean lung dose (MLD) $\geq 19.75$ had higher adenocarcinoma histology ( $79 \%$ vs $21 \%, \mathrm{p}=0.016)$, 
International Journal of Hematology and Oncology

\begin{tabular}{|c|c|c|c|c|}
\hline Variables & Response-3 & Response-6 & SUVmaxbase & SUVmean3 \\
\hline Response-1 & $\begin{array}{r}{[+] p<0.001} \\
r=0.549\end{array}$ & & & \\
\hline Response-3 & & $\begin{array}{r}{[+] p<0.001} \\
r=0.636\end{array}$ & & \\
\hline Tumour size & & & $\begin{aligned} {[+] p } & =0.010 \\
r & =0.371\end{aligned}$ & \\
\hline CTV & & & $\begin{aligned} {[+] p } & =0.018 \\
r & =0.383\end{aligned}$ & \\
\hline TLG & & & $\begin{aligned} {[+] p } & =0.015 \\
r & =0.446\end{aligned}$ & \\
\hline SUVmeanbase & & & $\begin{aligned} {[+] p } & =0.001 \\
r & =0.992\end{aligned}$ & \\
\hline KPS & & & $\begin{array}{r}{[-] p=0.037} \\
r=0.306\end{array}$ & \\
\hline SUVmax3 & & & & $\begin{array}{r}{[+] p<0.001} \\
r=0.997\end{array}$ \\
\hline
\end{tabular}

larger CTV ( $\geq 784 \mathrm{cc}, 68 \%$ vs $32 \%, \mathrm{p}=0.018)$ and larger MTV $(\geq 29,77 \%$ vs $23 \%, p=0.043)$.

The variables that positively affect survival according to univariate analysis were age of $<60$ years, KPS of $\geq 90$, gross tumour volume (GTV) of $<181$ cc, response- 6 , concomitant CHE, and administration of CHE for OS and age of $<60$ years, absence of metastases at diagnosis, a GTV of $<181 \mathrm{cc}$, presence of pathologic response and administration of CHE for PFS ( $\mathrm{p}<0.05$ ) (Table 4). Absence of metastasis at diagnosis, CTV of $<784$ cc and adjuvant CHE cycles of $<3$ had borderline significance for survival $(\mathrm{p}=0.05)$. In patients with SUV meanbase and SUVmean3 values of less than the median value, there was a trend towards improved survival rates ( $p>0.05)$. According to the Cox regression analysis employing the backward stepwise method, for each 1 unit increase, SUV meanbase (HR 1.18, 95\% CI: 1.01-1.38) and SUVmean3 (HR: 2.65, 95\% CI: 1.24-5.66) were found to be poor PFs for OS, whereas SUV mean3 (HR: 2.01, 95\% CI: 1.02-3.93) was found to be a poor PF for PFS.

\section{DISCUSSION}

The importance of response assessment in prognosis prediction and treatment management has been demonstrated in patients with locally advanced NSCLC. ${ }^{4-6}$ Response is known to change based on treatment type and RT dose. In a prospective study, 5-year locoregional PFS (LRPFS) for 67, 80 and 97 Gy was reported to be $12 \%, 35 \%$ and $49 \%$, whereas 5-year OS was reported to be $4 \%, 22 \%$ and $28 \%$, respectively. ${ }^{22}$ In the randomised study by Curran et al., significantly higher OR rates ( $70 \%$ vs $61 \%$ vs $65 \%$, respectively) and 5-year OS rates (16\% vs $10 \%$ vs $13 \%$, respectively) were reported for the concomitant 60 Gy chemo-RT regimen in comparison with sequential 63 Gy chemo-RT or concomitant hyperfractionated 69.6 Gy chemo-RT. ${ }^{2}$

Nodal response subsequent to neoadjuvant therapy is known to be important in terms of eligibility for surgery in patients with potentially resectable cancer. ${ }^{23}$ Randomised studies have shown a pCR of $10 \%$ and an OS benefit of 5\% with neoadjuvant CHE (1). In the retrospective study by Higgins et al., the mediastinal $\mathrm{pCR}$ in patients received neoadjuvant CHE or chemo-RT was $35 \%$ and $65 \%$, respectively, and $\mathrm{pCR}$ was found to be associated 
International Journal of Hematology and Oncology

\begin{tabular}{|c|c|c|c|c|}
\hline $\begin{array}{l}\text { Significance in } \\
\text { univariate analysis }\end{array}$ & Median OS, months $(95 \% \mathrm{Cl})$ & $\mathbf{p}$ & Median PFS, months (95\% Cl) & $\mathbf{p}$ \\
\hline Age & 54 (28.17-79.82), 5-year 40\% & 0.037 & 35 (5.87-64.12), 5-year 38\% & 0.025 \\
\hline$<60$ (n: 30) & Mean 66.10 (49.70-82.51) & & Mean 62.47 (45.92-79.02) & \\
\hline$\geq 60$ (n: 21) & $44.08(32.65-55.51)$ & & $32.80(19.91-45-69)$ & \\
\hline KPS & & 0.017 & & 0.079 \\
\hline$\geq 90$ (n: 37) & $60(24.42-95.57)$ & & $52(38.46-65.70)$ & \\
\hline$<90(\mathrm{n}: 14)$ & $35(9.28-60.71)$ & & $30(16.38-44.73)$ & \\
\hline Metastasis at diagnosis & & 0.059 & & 0.028 \\
\hline Present (n: 3) & $23(11.79-34.20)$ & & 15 (6.99-23.00) & \\
\hline Absent (n: 48) & $58(40.99-75.00)$ & & $40(5.18-74.81)$ & \\
\hline GTV, median & & 0.020 & & 0.021 \\
\hline$<181$ cc (n: 24) & Mean 67.44 (51.99-81.88) & & Mean $62(46.56-77.45)$ & \\
\hline$\geq 181$ cc (n: 20) & $40.76(23.66-57.66)$ & & $39(22.13-57.45)$ & \\
\hline CTV, median & & 0.057 & & 0.093 \\
\hline$\geq 784$ cc (n: 22) & Mean 44 (28.88-60.62) & & Mean 42 (26.28-59.32) & \\
\hline$<784$ cc (n: 20) & $66(39.29-83.04)$ & & $59(40.54-77.99)$ & \\
\hline Response- 6 & & 0.020 & & 0.45 \\
\hline Complete (n: 11) & $80(44.2-115.47)$ & & $60(39.35-81.50)$ & \\
\hline Partially (n: 10) & $58(48.00-67.99)$ & & 57 (35.96-79.62) & \\
\hline Stable/progression (n: 6) & $27(1.55-52.44)$ & & $39(0.25-78.74)$ & \\
\hline Pathological response & & 0.083 & & 0.040 \\
\hline Present (n: 7) & Mean 69 (42.95-95.04) & & Mean 68 (40.40-95.76) & \\
\hline Absent (n. 4) & $37(13.13-62.36)$ & & $20(7.24-33.75)$ & \\
\hline Concurrent $\mathrm{CHE}$ & & 0.019 & & 0.091 \\
\hline Present (n: 15) & $60(50.46-69.53)$ & & $43(0.00-88.64)$ & \\
\hline Absent (n: 36) & $27(8.60-45.39)$ & & $16(7.37-24.62)$ & \\
\hline Total CHE & & 0.002 & & 0.027 \\
\hline Present (n: 47) & $58(40.51-75.48)$ & & $40(10.89-69.10)$ & \\
\hline Absent (n: 4) & $10(3.14-16.86)$ & & $6(2.08-9.92)$ & \\
\hline Adjuvant $\mathrm{CHE}$ cycles & & 0.057 & & 0.058 \\
\hline$\geq 3$ (n: 23) & Mean 48 (35.24-61.90) & & Mean 36 (19.56-53.87) & \\
\hline$<3(\mathrm{n}: 6)$ & $79(60.08-98.91)$ & & $71(46.99-96.33)$ & \\
\hline SUV meanbase, median & & 0.30 & & 0.28 \\
\hline$\geq 9,37(\mathrm{n}: 15)$ & $28(11.49-44.50)$ & & $16(5.64-26.35)$ & \\
\hline$<9,37(\mathrm{n:} 14)$ & 54 (28.29-79.70) & & 35 (15.98-64.01) & \\
\hline SUVmean3, median & & 0.97 & & 0.95 \\
\hline$\geq 2,81$ (n: 11) & $39(0 ., 00-82.36)$ & & $17(0.00-53.2)$ & \\
\hline$<2,81$ (n: 9) & $58(0.00-122.13)$ & & $18(0.00-40.93)$ & \\
\hline $\begin{array}{l}\text { Significance in Cox } \\
\text { regression analysis }\end{array}$ & OS, HR (95\% Cl) & & PFS, HR $(95 \% \mathrm{Cl})$ & \\
\hline SUVmeanbase, numeric & HR: 1.18 (1.01-1.38) & 0.033 & HR: 1.17 (0.99-1.37) & 0.053 \\
\hline SUVmean3, numeric & HR: 2.65 (1.24-5.66) & 0.012 & HR: 2.01 (1.02-3.93) & 0.041 \\
\hline
\end{tabular}


with disease-free survival (DFS) and LC. ${ }^{24}$ In the INT 0159 study that included 402 patients who had $\mathrm{pN} 2$ disease, the patients were randomised to surgery or curative RT arms following 45 Gy chemoRT. ${ }^{25}$ The 5-year OS for patients with pN0, persistent $\mathrm{N} 2$ disease and those administered curative chemo-RT was $41 \%, 24 \%$ and $20 \%$, respectively; and the importance of nodal response was demonstrated for determining the patients who are eligible for surgery. In this study, pCR was achieved in $46 \%$ of the patients with stable response, and it should be noted that anatomical response assessment based on CT is insufficient.

Currently, diameter-based anatomical response assessment (RECIST) has been replaced by metabolic response assessment (PERCIST). ${ }^{16}$ In the NEOSCAN study, a change of $<35 \%$ in SUV peak of patients treated with 2 cycles of neoadjuvant $\mathrm{CHE}$ was found to be predictive and prognostic for CHE regimen change. ${ }^{26}$ Lee et al. reported that in 44 patients received neoadjuvant CHE, the time to recurrence was prolonged with a radiological response of $\geq 30 \%$ and a SUV max response of $\geq 25 \%$ (49 vs 23 months). ${ }^{27}$ The accuracy of pathologic response prediction was reported to be $70 \%$ with radiological evaluation, 52 to $75 \%$ with metabolic evaluation and 73 to $82 \%$ with both evaluation.

In a study by Iravani et al. that included 87 patients treated with RT or chemo-RT, it was reported that the PET/CT response at 3rd month after treatment was associated with OS. ${ }^{28}$ Conversely, Chen et al. evaluated 25 patients at week 5 during chemo-RT (at a median of $46 \mathrm{~Gy}$ ) and 3rd month after chemoRT and reported that TLG (cut-off 65\%) and MTV (cut-off $42 \%$ ) changes during RT resulted in significantly increased OS and PFS but these changes were not significant at $3 \mathrm{rd}$ month. ${ }^{29}$ It has been shown that evaluation using PET/CT can led to a dose increase or treatment modification during the treatment and it has also been found to provide $3 \%$ benefit in terms of salvage therapy for patients who progress 3 months after the treatment. ${ }^{12,30,31}$

Because RT causes inflammatory reactions in lung tissues, there is an ongoing discussion about the best time to evaluate response using PET/CT. ${ }^{32,33}$ Choi et al. underlined that the biological lethal damage to tumour cells occurred 8-12 weeks after $\mathrm{RT}$; however, this could not be beneficial in terms of treatment modification and LC. ${ }^{34}$ In a study by Massaccesi et al., the median tumour and nodal SUVmax values at the baseline, at week 3 during treatment and at 1 month after chemo-RT were 16.1 and 10, 4.7 and 7.7, 4.6 and 2.1, respectively. ${ }^{35}$ It was reported that the response started during the treatment (76\% OR) and continued in month 1 after treatment (95\% OR, 38\% CR), it was correlated with the total dose and the DFS significantly increased with metabolic $\mathrm{CR}$ at month 1 . On the other hand, it was emphasized that response evaluation would be challenging up to 6-24 months due to persistent hypermetabolism in patients with early stage lung cancer treated with stereotactic body RT. ${ }^{36}$ Limited resolution, difficulties in determining margins of lymph nodes (conglomerate or adjacent to tumour), high rates of FP and lack of a consensus concerning which stations should be assessed were reported to be the reasons for nodal response accuracy being lower in PET/CT., ${ }^{9,16,19,34,37}$ Arnett et al. could not demonstrate any significant correlation between nodal PET parameters and nodal pCR. ${ }^{38}$ Contrarily, Okazaki et al. reported that both tumour and nodal TLG were significant in terms of LC and disease-specific survival. ${ }^{39}$ In our study, nodal PET/CT parameters were not assessed due to the difficulties in measurement. At month 3, OR was observed to be $76 \%$ in PET/CT evaluation and the correlation between metabolic parameters and responses indicated that the response continued for up to 6 months.

It is still debatable whether SUV max is the best prognostic marker in response evaluation. ${ }^{15,20,33}$ Van Loon et al. reported that each 1\% decrease in primary tumour and nodal SUV max following $1 \mathrm{cy}$ cle of CHE resulted in a $2 \%$ increase in OS (40). In the study by Huang et al. the significant cut-off values with 40 Gy chemo-RT were found to be $42 \%$ for SUVmean (37.5 vs. 19.5 months) and $30 \%$ for MTV (36.5 vs. 16 months) in terms of median OS. ${ }^{41}$ In the study by Van Diessen et al. there were no OS differences based on baseline PET parameters, whereas both intensity (SUVmax, SUVmean) and volumetric (TLG, MTV) parameters were correlated with OS at month 1 after treatment. ${ }^{42}$ In the ACRIN 6668/RTOG 0235 study, it was found that the baseline SUVpeak (10.3) and SUVmax (13.1) were not significant for OS; however, each 1-point increase in SUVpeak3 increased the mortality risk 
by $9 \% .^{20}$ In the 2015 analysis of the study, a nodal residue SUV max of $>5$ and change of $<25 \%$ were found to be unfavourable in terms of locoregional control. ${ }^{37}$ In the 2017 analysis of the study, it was also emphasised that MTV and TLG were promising biomarkers for locoregional control and could be used in routine practice for prognostic classification. ${ }^{33}$ In the present study, each 1 unit increase in SUVmeanbase and SUVmean3 values was found a significantly poor PF for survival. The lack of a significant correlation between MTV, TLG, and survival was believed to be associated with the small number of patients evaluated for these parameters.

Age, sex, KPS, stage, total RT dose, fraction dose, administration of CHE, targeted volumes and risky organ doses are known to be PFs in terms of complications and survival in patients with NSCLC. ${ }^{1}$ The rates of severe RP are range from 10 to $20 \%$, which is associated with an MLD of $>20$ Gy in patients treated with chemo-RT. ${ }^{43}$ Liao et al. reported that although GTV was positively correlated with $\geq$ G3 RP, increased OS was observed owing to decreased lung V20 dose in 496 patients who were administered 63 Gy chemo-RT with IMRT. ${ }^{44}$ In our previous study that included 68 patients, it was found that the therapeutic dose range in where MLD could be kept under 20 Gy with significant survival benefit was between $>59.4$ Gy and $\leq 63$ Gy. ${ }^{45}$ In the study by Warner et al. 180-day early mortality rate was shown to be lower with a GTV of $<100 \mathrm{cc}^{.46}$ In the current study, age, KPS, absence of metastases at diagnosis, administration of CHE, concomitant CHE, response- 6 and pathologic response were found to be significant in terms of survival consistent with the literature. Increased $\mathrm{RP}$ rates among patients who were administered a fraction dose of $<200 \mathrm{cGy}$ can be indirectly attributed to increased CTV and MLD. Conversely, patients with RP may have significantly increased OR at month 3 , which could have indirectly affected survival positively. A tumour GTV of $<181 \mathrm{cc}$ was found to be a significantly favourable PF for OS and PFS.

The positive aspect of the study was that it had a long follow-up period. The limitations were as follows: the study was retrospective, evaluation of PET/CT parameters was conducted with small number of patients, $41 \%$ of the patients were ad- ministered neoadjuvant CHE and not all of these patients were assessed using PET/CT prior to RT; moreover, it could not be determined whether the use of targeted agents as part of maintenance therapy had any effect on survival.

\section{Conclusion}

PET/CT parameters can be used as beneficial markers in terms of prognosis, treatment modification and survival in patients with NSCLC who are administered curative chemo-RT. Although imaging with PET/CT was found to be significant in terms of survival at 3 months after the treatment, it can also be a disadvantage owing to the RT-induced changes and the inability to differentiate residual tumours. It is believed that early assessment during and after the treatment can be advantageous in terms of treatment modification.

\section{REFERENCES}

1. Rengan R, Chetty IJ, Decker R, Langer CJ, O'Meara WP and Movsas B (eds). Lung cancer. In: Halperin EC, Wazer DE, Perez CA, Brady LW, eds. Principles and Practice of Radiation Oncology, 6th ed. Philadelphia: LWW, 2013: 973-995.

2. Curran WJ Jr, Paulus R, Langer CJ, et al. Sequential vs. concurrent chemoradiation for stage III non-small cell lung cancer: randomized phase III trial RTOG 9410. J Natl Cancer Inst 103: 1452-1460, 2011.

3. Auperin A, Le Pechoux C, Rolland E, et al. Meta-analysis of concomitant versus sequential radiochemotherapy in locally advanced non-small-cell lung cancer. J Clin Oncol 28: 2181 2190, 2010.

4. Perez CA, Stanley K, Rubin P, et al. A prospective randomized study of various radiation doses and fractionation schedules in the treatment of inoperable non-oat-cell carcinoma of the lung: Pre-liminary report by the Radiation Therapy Oncology Group. Cancer 45: 2744-2753, 1980.

5. Langendijk HA, Lamers RJ, ten Velde GP, et al. Is the chest radio-graph a reliable tool in the assessmant of tumour response after radiotherapy in nonsmall cell lung carcinoma? Int J Radiat Oncol Biol Phys 41: 1037-1045, 1998.

6. Werner-Wasik M, Xiao Y, Pequignot E, et al. Assessment of lung cancer re-sponse after nonoperative therapy: tumour diameter, bidimensional product, and volume. A serial CT scan-based study. Int J Radiat Oncol Biol Phys 51: 56-61, 2001.

7. Warburg O. On the origin of cancer cells. Science 123: 309314, 1956.

8. Pieterman RM, van Putten JW, Meuzelaar JJ, et al. pre-operative staging of non-small-cell lung cancer with positronemission tomography. N Engl J Med 343: 254-261, 2000. 
9. Lardinois D, Weder W, Hany TF, et al. Staging of non-smallcell lung cancer with integrated positron-emission tomography and computed tomograpy. N Eng J Med 348: 25002507, 2003.

10. Gould MK, Kuschner WG, Rydzak CE, et al. Test performance of positron emission tomography and computed tomography for mediastinal staging in patients with nonsmall-cell lung cancer: a meta-analysis. Ann Intern Med 139: 879-892, 2003.

11. Mac Manus MP, Hicks RJ, Matthews JP, et al. Positron emission tmograpy is superior to computed tomography scanning for response-assessment after radical radiotherapy or chemoradiotherapy in patients with non-small-cell lung cancer. J Clin Oncol 21: 1285-1292, 2003.

12. Bradley J, Bae K, Choi N, et al. A phase II comparative study of gross tumour volume definition with or without PET/CT fusion in dosimetric planning for non-small-cell lung cancer (NSCLC): primary analysis of Radiation Therapy Oncology Group (RTOG) 0515. Int J Radiat Oncol Biol Phys 82: 435441, 2012.

13. Ung YC, Gu CS, Cline K, et al. An Ontario Clinical Oncology Group (OCOG) randomized trial of FDG PET/CT in patients with stage 3 non-small cell lung cancer (NSCLC): impact of PET on radiation treatment volumes. Abstract presented at the 14th World Conference on Lung Cancer; 2011, J Thorac Oncol, 6 (6), Suppl. 2.

14. de Cabanyes Candela S, Detterbeck FC. A systematic review of restaging after induction thera-py for stage Illa lung cancer: prediction of pathologic stage. J Thorac Oncol 5: 389-398, 2010.

15. Na F, Wang J, Li C, et al. Primary tumour standardized uptake value meas-ured on F18-Fluorodeoxyglucose positron emission tomography is of prediction value for survival and local control in non-small-cell lung cancer receiving radiotherapy: meta-analysis. J Thorac Oncol 9: 834-842, 2014.

16. Wahl RL, Jacene H, Kasamon $\mathrm{Y}$, Lodge MA. From RECIST to PERCIST: Evolving consider-ations for PET response criteria in solid tumors. J Nucl Med 50 (Suppl 1): 122S-150S, 2009.

17. Cerfolio RJ, Bryant AS, Winokur TS, et al. Repeat FDGPET after neoadju-vant therapy is a predictor of pathologic response in patients with non-small cell lung cancer. Ann Thorac Surg 78: 1903-1909, 2004.

18. Eschmann SM, Friedel G, Paulsen F, et al. Repeat 18F-FDG PET for monitoring neoadjuvant chemotherapy in patients with stage III non-small cell lung cancer. Lung Cancer 55: 165-171, 2007.

19. Eschmann SM, Friedel G, Paulsen F, et al. 18F-FDG PET for assessment of therapy response and preoperative reevaluation after neoadjuvant radio-chemotherapy in stege III non-small cell lung cancer. Eur J Nucl Med Mol imaging 34: 463-471, 2007.

20. Machtay M, Duan F, Siegel BA, et al. predicition of survival by [18F] Fluorodeoxyglucose positron emission tomography in patients with locally ad-vanced non-small-cell lung cancer undergoing definitive chemoradiation therapy: results of the ACRIN 6668/RTOG 0235 trial. J Clin Oncol 31: 3823-3830, 2013.

21. Common Terminology Criteria for Adverse Events v4. https://evs.nci.nih.gov/ftp1/CTCAE/CTCAE_4.03/CTCAE_607 4.03_2010-06-14_QuickReference_8.5x11.pdf

22. Kong FM, Haken RKT, Schipper MJ, et al. High-dose radiation improved local tumour control and overall survival in patients with inopera-ble/unresectable non-small-cell lung cancer: long-term results of a radiation dose escalation study. Int J Radiat Oncol Biol Phys 63: 324-333, 2005.

23. Martini N, Kris MG, Flehinger BJ, et al. Preoperative chemotherapy for stage Illa (N2) lung cancer: the Sloan-kettering experience with 136 patients. Ann Thorac Surg 55: 1365-1373, 1993.

24. Higgins K, Chino JP, Marks LB, et al. Preoperative chemotehrapy versus preoperative chemoradiotherapy for stage III (N2) non-small-cell lung cancer. Int J Radiat Oncol Biol Phys 75: 1462-1467, 2009 .

25. Albain KS, Swann RS, Rusch WW, et al. Radiotherapy plus chemotherapy with or without surgical resection for stage III non-small-cell lung cancer: a phase III randomised controlled trial. Lancet 374: 379-386, 2009.

26. Chaft JE, Dunphy M, Naidoo J, et al. Adaptive neoadjuvant chemotherapy guided by (18)F-FDG PET in resectable nonsmall cell lung cancers: The NE-OSCAN trial. J Thorac Oncol 11: 537-544, 2016.

27. Lee HY, Lee HJ, Kim YT, et al. Value of combined interpretation of computed tomography response and positron emission tomography response for predic-tion of prognosis after neoadjuvant chemotherapy in non-small cell lung cancer. J Thorac Oncol 5: 497-503, 2010.

28. Iravani A, Turgeon GA, Akhurst T, et al. PET-detected pneumonitis following curative-intent chemoradiation in non-small cell lung cancer (NSCLC): rec-ognizing patterns and assessing the impact on the predictive ability of FDG-PET/CT response as-sessment. Eur J Nucl Med Mol Imaging 46: 18691877,2019

29. Chen HHW, Su WC, Guo HR, et al. Prognostic value of volumetric metabolic parameter changes determined by during and after radiotherapy-based 18F-FDG PET/CT in stage III non-small cell lung cancer. Kaohsiung J Med Sci 35: 151159, 2019.

30. van Elmpt W, De Ruysscher D, van der Salm A, et al. The PET-boost randomised phase II dose-escalation trial in nonsmall cell lung cancer. Radiother Oncol 104: 67-71, 2012.

31. van Loon J, Grutters J, Wanders R, et al. Follow-up with 18FDG-PET-CT after radical radiotherapy with or without chemotherapy allows the detection of potentially curable progressive disease in non-small cell lung cancer patients: a prospective study. Eur J Cancer 45: 588-595, 2009. 
32. Allen-Auerbach $M$, Weber WA. Measuring response with FDG-PET: methodological aspects. Oncologist 14: 369-377, 2009.

33. Salavati A, Duan F, Snyder BS, et al. Optimal FDG PET/CT volumetric parameters for risk stratification in patients with locally advanced non-small cell lung cancer: results from the ACRIN 6668/RTOG 0235 trial. Eur J Nucl Med Mol Imaging 44: 1969-1983, 2017.

34. Choi NC, Fischman AJ, Niemierko A, et al. Dose-response relation-ship between probability of pathologic tumour control and glucose metabolic rate measured with FDG PET after preoperative chemoradiotherapy in locally advanced nonsmall-cell lung cancer. Int J Radiat Oncol Biol Phys 54: 102435, 2002.

35. Massaccesi M, Calcagni ML, Spitilli MG, et al. 18F-FDG PETCT during chemo-radiotherapy in patients with non-small cell lung cancer: the early metabolic response correlates with the delivered radiation dose. Radiat Oncol 7: 106, 2012.

36. Zhang X, Liu H, Balter P, et al. Positron emission tomography for assessing local failure after stereotactic body radiotherapy for non-small-cell lung cancer. Int J Radi-at Oncol Biol Phys 83: 1558-1565, 2012.

37. Markovina S, Duan F, Snyder BS, et al. Regional lymph node uptake of [(18)F] Fluorodeoxyglucose after definitive chemoradiation therapy predicts local-regional failure of locally advanced non-small cell lung cancer: Results of ACRIN 6668/ RTOG 0235. Int J Radiat Oncol Biol Phys 93: 597-605, 2015.

38. Arnett AL, Packard AT, Mara K, et al. FDG-PET parameters as predictors of pathologic response and nodal clearance in patients with stage III non-small cell lung cancer receiving neoadjuvant chemoradiation and surgery. Pract Radiat Oncol 7: e531-e541, 2017.

39. Okazaki E, Seura H, Hasegawa $Y$, et al. Prognostic value of the volumetric parameters of dual-time point 18F-FDG PET/ CT in non-small cell lung cancer treated with definitive radiation therapy. Am J Roentgenol 213: 1366-1373, 2019.

40. van Loon J, Offermann C, Ollers M, et al. Early CT and FDGmetabolic tumour volume changes show a significant correlation with survival in stage I-III small cell lung cancer: a hypothesis generating study. Radiother Oncol 99: 172-175, 2011.

41. Huang $\mathbf{W}$, Fan $M$, Liu B, et al. Value of metabolic tumour volume on repeated 18F-FDG PET/CT for early prediction of survival in locally advanced non-small cell lung cancer treated with concurrent chemoradiotherapy. J Nucl Med 55: 15841590, 2014.

\section{International Journal of Hematology and Oncology}

42. van Diessen JNA, La Fontaine M, van den Heuvel MM, et al. Local and regional treatment response by 18FDG-PETCT-scans 4 weeks after concurrent hypofractionated chemoradiotherapy in locally advanced NSCLC. Radiother Oncol S0167-8140(19)33144-5, 2019.

43. Marks LB, Bentzen SM, Deasy JO, et al. Radiation dosevolume effects in the lung. Int J Radiat Oncol Biol Phys 76 (3 Suppl): S70-76, 2010.

44. Liao ZX, Komaki RR, Thames HD Jr, et al. Influence of technologic advances on outcomes in patients with unresectable, locally advanced non-small-cell lung cancer receiving concomitant chemoradiotherapy. Int J Radiat Oncol Biol Phys 76: 775-781, 2010.

45. Okumus D, Sarihan S, Gozcu S, Sigirli D. The relationship between dosimetric factors, side effects, and survival in patients with non-small cell lung cancer treated with definitive radiotherapy. Med Dosim 42: 169-176, 2017.

46. Warner A, Dahele M, Hu B, et al. Factors associated with early mortality in patients treated with concurrent chemoradiation therapy for locally advanced non-small cell lung cancer. Int $J$ Radiat Oncol Biol Phys 94: 612-620, 2016.

\section{Correspondence:}

\section{Dr. Sureyya SARIHAN}

Uludag Universitesi, Tip Fakultesi

Radyasyon Onkolojisi Anabilim Dali

16059 Gorukle kampusu, Nilufer

BURSA / TURKEY

Tel: (+90-224) 2953441

e-mail: ssarihan@uludag.edu.tr

\section{ORCIDs:}

Sureyya Sarihan

0000-0003-4816-5798

Gokce Belge Bilgin 0000-0001-8258-1774

Deniz Sigirli 